\title{
$\rho$-PROJECTIVE AND $\sigma$-INVOLUNTARY VARIATIONAL INEQUALITIES AND IMPROVED PROJECTION METHOD FOR PROJECTED DYNAMICAL SYSTEM
}

Siddharth Mitra ${ }^{1^{*}}$, Prasanta Kumar Das ${ }^{2}$

${ }^{1 *}, 2$ School of Applied Sciences (Mathematics), KIIT University, Bhubaneswar, Odisha, India. Email: ${ }^{1 *}$ siddharthmitra06@gmail.com, ${ }^{2}$ dasprasantkumar@yahoo.co.in

\author{
Article History: Received on $28^{\text {th }}$ March 2021, Revised on $26^{\text {th }}$ April 2021, Published on $6^{\text {th }}$ May 2021
}

\begin{abstract}
Purpose of study: To introduce the concept of projective and involuntary variational inequality problems of order $\rho$ and $\sigma$ respectively. To study the equivalence theorem between these problems. To study the projected dynamical system using self involutory variational inequality problems.
\end{abstract}

Methodology: Improved extra gradient method is used.

Main Finding: Using a self-solvable improved extra gradient method we solve the variational inequalities. The algorithm of the projected dynamical system is provided using the $\underline{\mathrm{RK}}-4$ method whose equilibrium point solves the involutory variational inequality problems.

Application of this study: Runge-Kutta type method of order 2 and 4 is used for the initial value problem with the given projected dynamical system with the help of self involutory variational inequality problems.

The originality of this study: The concept of self involutory variational inequality problems, projective and involuntary variational inequality problems of order $\rho$ and $\sigma$ respectively are newly defined.

Keywords: Involutory_Variational Inequalities, Projective Variational Inequalities, Projection Method, Projected Dynamical System.

\section{INTRODUCTION}

In a Banach space $\mathrm{X}$ with dual $X^{*}$, we say that an operator $\mathrm{A}: \mathrm{X} \rightarrow X^{*}$ is $\rho$-projective if its minimal polynomials $\rho^{-1} x^{2}-x$ for some $\rho \in R$ and an operator $B: X \rightarrow X$ is $\sigma$-involutory if its minimal polynomial equation is $\sigma^{-1} x^{2}-1$ for some $\sigma \in \mathrm{R}$. Now for any two Banach spaces $\mathrm{X}$ and $\mathrm{Y}$, if $\mathrm{f}: \mathrm{X} \rightarrow \mathrm{Y}$ and $\mathrm{g}: \mathrm{Y} \rightarrow \mathrm{X}$, the $g \circ f: X \rightarrow X$ and $f \circ g: Y \rightarrow Y$. If there exist maps

$\widetilde{f}: \mathrm{X} \rightarrow \mathrm{E} \subset \mathrm{R}$ and $\mathrm{p}_{X}: \mathrm{E} \rightarrow \mathrm{Y}$ such that $\mathrm{f}=\mathrm{p}_{X} \circ \tilde{f}$ is continuous, then $\widetilde{f}$ is called the lifting map of $\mathrm{f}$ and $\mathrm{p}_{X}$ is called the covering map. Similarly, if there exists maps $\tilde{g}: \mathrm{Y} \rightarrow \mathrm{E} \subset \mathrm{R}$ and $\mathrm{p}_{Y}: \mathrm{E} \rightarrow \mathrm{X}$ such that $\mathrm{g}=\mathrm{p}_{Y} \circ \widetilde{g}$ is continuous, then $\widetilde{g}$ is called the lifting map of $\mathrm{g}$ and $\mathrm{p}_{Y}$ is called the covering map. Hence if $\mathrm{R}(\tilde{f}) \cap \mathrm{R}(\tilde{g}) \neq \emptyset$,

$\mathrm{D}(\tilde{f}) \cap \mathrm{R}\left(\widetilde{\mathrm{p}_{Y}}\right) \neq \emptyset$ and $\left.\mathrm{D}(\tilde{g}) \cap \mathrm{R}\left(\widetilde{P_{X}}\right\}\right) \neq \emptyset$ where $\mathrm{D}(\mathrm{f})$ represents the domain of $\mathrm{f}$, then there exists at least one bijective map $\mathrm{h}: \mathrm{E} \rightarrow \mathrm{W}$ where $\mathrm{E}=\mathrm{R}(\tilde{f}) \cap \mathrm{R}(\tilde{g})$ and $\mathrm{W}=\mathrm{D}(\tilde{f}) \cap \mathrm{R}\left(\widetilde{p_{Y}}\right) \cap \mathrm{D}(\tilde{g}) \cap \mathrm{R}\left(\widetilde{p_{X}}\right)$ are of finite and same dimension. Siddiqi, A. H., Ansari, Q. H., \& Khaliq, A. (1995), Solodov, M. V., \&Svaiter, B. F. (1997), Thong, D. V., \& Cho, Y. J. (2020).

\section{LITERATURE REVIEW}

In the recent decades, the theory of variational inequality is used to solve various types of inequality, and equilibrium problems arises in the branches of Engineering, Physical Sciences, Applied Mathematics, Finance, Medical, and so on. In fact, the problem are can be expressed in the form of variational inequality problems (VIP) which is introduced by Stampacchia, G. (1964). Later the authors have defined the variational inequality problems in vector spaces, Haudarff topological spaces, and H-spaces. For reference, we refer Behera, A., \& Panda, G. K. (1997), Das P. K., \& Mohanta, S.

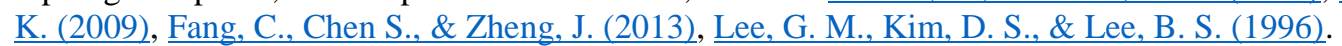

Various authors have studied the theory of variational inequalities using the projection method. Solodov and Svaiter (1997) have developed the improved projection method to solve the variational inequalities. Nagurney, A., \&Zhang, D. $\underline{(1996)}$ have discussed the equilibrium solution of a projected dynamical system using the projective operator. Let $F: K$ $\subset X \rightarrow X^{*}$ be a linear mapping and the pairing $\langle f, x\rangle$ denotes the value $f \in X^{*}$ of at $x \in K$. The positive orthant $K$ and normal cone $N$ are defined by $K^{+}=\left\{f \in X^{*}:\langle f, x\rangle \geq 0, x \in K\right\}$ and $N=\left\{g \in X^{*}:\langle g, x\rangle \leq 0, x \in K\right\}$. Bardaro, C., \& Ceppitelli, R. (1988), Behera, A., Das, P. K. (2006), Giannessi, F. (1980), Limaye, B. V. (1997), Malitsky, Y. (2015).

The variational inequality problems (VIP) is to find $x^{*} \in K$ such that

$\left\langle F\left(x^{*}\right), x-x^{*}\right\rangle \geq 0$ for all $x \in K$

where the directed feasible set $K^{+}(x)$ of the solution of VIP is defined by 
$K^{+}(x)=\left\{x^{*} \in K:\left\langle F(x *), x-x^{*}\right\rangle \geq 0, x \in K\right\}$.

The dual variational inequality problems (DVIP) is to find $x^{*} \in K$ such that

$\left\langle F(x), x-x^{*}\right\rangle \geq 0$ for all $x \in K$

(DVIP)

where directed feasible set $K^{-}(x)$ of the solution of DVIP is defined by

$K^{-}(x)=\left\{x^{*} \in K:\left\langle F(x), x-x^{*}\right\rangle \leq 0\right.$ for all $\left.x \in K\right\}$.

\section{Projection operator and its application:}

A map $P: X \rightarrow X$ is a projection map if it satisfies $P^{2}=P$, i.e., $P(x)=0$ or $(I-P)(x)=0$ for all $x \in X$. The zero space of $P$ is defined by

$Z(P)=\{x \in X: P(x)=0\}$,

and the range space of $P$ is defined by

$R(P)=\{x \in X: P(x)=x\}$.

In fact, $X=Z(P) \oplus R(P)$, i.e., $X=Z(P) \cup R(P)$ and $Z(P) \cap R(P)=\emptyset$. Let $X$ be a Hilbert space and $K$ be a subset of $X$. For our study, we denote $f: X \rightarrow X$ as a fixed point function if $f(x)=x$ for at least one $x \in X$. Let $\mathrm{F}$ be the set of fixed-point functions. For any $f \in \mathrm{F}$ there exists an unique $x \in X$ such that $f(x)=x$. Now the approximation problem (AP) is to minimize the function $g: X \rightarrow \mathrm{R}$ defined by $g(\mathrm{x})=\|\mathrm{x}-\mathrm{z}\|$ for all $z \in K$ for each $x \in K \subset X$ the Hilbert space. If for each $x$ $\in K$, there exists an unique $y \in K$ such that $\|x-y\|=\min _{z \in X}\|x-z\|$, then the point $y \in K$ is called the projection of $x$ on $K$ and written as $y=\mathbb{P}_{K}(x)$. Das, P.K. (2011), He, B. S., \& Liao, L. Z. (2002), Mosco, U. (1972).

\section{Definition}

The projection operator $\mathbb{P}_{K}: K \rightarrow K$ is non-expansive, i.e., $\left\|\mathbb{P}_{K}\left(x_{1}\right)-\mathbb{P}_{K}\left(x_{2}\right)\right\| \leq\left\|x_{1}-x_{2}\right\|$ for all $x_{1}, x_{2} \in K$, i.e., $\mathbb{P}_{K} \in \mathrm{F}$, implying $\mathbb{P}_{K}$ is continuous on $K$ and has a fixed point in $K$. It is obvious that $\mathbb{P}_{K}(x)=x$ for all $x \in K$ and holds the following result. If $K$ is a closed set in $X$, then for each $x \in K$, there exists an unique $y \in K$ such that $y=\mathbb{P}_{K}(x)$, i.e., $\quad \| x$ $-y\left\|=\min _{z \in X}\right\| x-z \|$, which can be written as a problem to find an unique $y \in K$ such that $\langle y, z-y\rangle \geq\left\langle x, z^{-} y\right\rangle$ for all $z \in K$. This is a particular representation of the variational inequality problem where $F(x)=y-x$.

\section{METHODOLOGY}

\section{f-Projection Method}

Let $f: K \rightarrow K$ be a Lipschiz continuous function on $X$. For our need, we define the concept of projection functional operator as follows:

Definition: Let $f: X \rightarrow X$ be any linear function. If for each $x \in K$, there exists an unique $y \in K$ such that

$\|x-y\|=\min _{z \in X}\|f(x)-z\|$

The point $y \in K$ is called the $f$-projection of $x$ on $K$ and written as

$y=\mathbb{P}_{K}(f(x))=\mathbb{P}_{K}^{f}(x)$

It is obvious that $\mathbb{P}_{K}^{f}(x)=x$ if $x \in K$ is the fixed point of $f$.

Definition: The projection functional operator $\mathrm{P}_{K}^{f}$ :ran $(f) \rightarrow \operatorname{ran}(f)$ is non-expansive on $K$, i.e.,

$\left\|\mathbb{P}_{K}^{f}\left(x_{1}\right)-\mathbb{P}_{K}^{f}\left(x_{2}\right)\right\| \leq\left\|f\left(x_{1}\right)-f\left(x_{2}\right)\right\|$

for all $x_{1}, x_{2} \in K$, implying $\mathbb{P}_{K}^{f}$ is continuous on $\operatorname{ran}(f) \subset K$ and has a fixed point in $K$.

Theorem: Let $f: K \rightarrow X$ Lipschitz continuous on the closed convex set $K$. Then there exist a $y \in K$ such that

$\langle\mathrm{y}, \mathrm{z}-\mathrm{y}\rangle \geq\langle\mathrm{f}(\mathrm{x}), \mathrm{z}-\mathrm{y}\rangle$ for all $z \in K$

Proof: Since $f: K \rightarrow X$ Lipschitz continuous function on $K$,

for some rank $L>0$. Since $K$ is closed, taking $F(x)=x+f(x)-y \in X$ is nearest to an unique $y \in K$ for each $x \in K$ for some $F: K \rightarrow X$, we have

$\langle y, z-y\rangle \geq\langle F(x), z-y\rangle$ for all $z \in K$, i.e.,

$\langle y, z-y\rangle \geq\langle x+f(x)-y, z-y\rangle$

for all $z \in K$, implying $y=\mathbb{P}_{K}^{f}(x)$. Again since $\mathbb{P}_{K}^{f}$ is non-expansive on $K$, we have,

$\left\|\mathbb{P}_{K}^{f}\left(x_{1}\right)-\mathbb{P}_{K}^{f}\left(x_{2}\right)\right\| \leq\left\|f\left(x_{1}\right)-f\left(x_{2}\right)\right\| \leq L\left\|x_{1}-x_{2}\right\|$ 
for all $x_{1}, x_{2} \in K$. For $L \in(0,1], \mathbb{P}_{K}^{f}$ has a fixed point, implying $f$ has a fixed point on $K$.

Let $x^{*}=y^{*}$ be the fixed point of $f$, then $f\left(x^{*}\right)=x^{*}=y^{*}$, we get

$\left\langle y^{*}, z^{-}-y^{*}\right\rangle \geq\left\langle x^{*}+f\left(x^{*}\right)-y^{*}, z-y^{*}\right\rangle=\left\langle x^{*}, z-y^{*}\right\rangle=\left\langle f\left(x^{*}\right), z-y^{*}\right\rangle$

for all $z \in K$ where $y^{*}$ is the unique point corresponds to $x^{*}$. This completes the proof.

Definition: For $x \in K$ and $v \in X$, the $f$-projection of the vector $v$ at $x$ (with respect to $K$ ) is defined by

$\prod_{K}^{f}(x ; v)=\lim _{\lambda \rightarrow 0} \frac{\mathbb{P}_{K}^{f}(x+\lambda v)-x}{\lambda}$.

\section{o-Involutory Variational Inequality Problems and Projection Method}

Let $X$ be a Banach space. We consider a class of map $A: X \rightarrow X$ satisfying the condition $A^{3}=\sigma A$ for some $\sigma \in \mathrm{R}$. In this case, we have either $A(\mathrm{x})=0$ or $\left(A^{-1}-\sigma^{-1} \mathrm{~A}\right)(\mathrm{x})=0$, i.e $\left(A^{-1}-\sigma^{-1} \mathrm{~A}\right)(\mathrm{x})=0$ for all $x \in X$. For simplicity, we denote the operator $A_{\sigma, 2}=A_{2}(; \sigma)=\sigma^{-1} A^{2}$ and $A_{\sigma, 2}=I-A_{2}(; \sigma)=A^{-1}-\sigma^{-1} \mathrm{~A}$. Thus for each $x \in X, A_{\sigma, 2}(x)=A_{2}(; \sigma)(X)=\left(I-A_{2}(; \sigma)\right)(x)$ $=x-A_{2}(x ; \sigma)=\left(A^{-1}-\sigma^{-1} \mathrm{~A}\right)(x)$.

For $\sigma=1$, we have $A_{1,2}=A^{2}$. Therefore $A_{\sigma, 2}=\sigma^{-1} \mathrm{~A}$ and $\mathrm{A}_{\sigma, 2}=I-A_{\sigma, 2}$.

The zero space of $A$ and the range space of $A$ are defined by

$Z(A)=\{x \in X: A(x)=0\}$ and $R(A)=\left\{y \in X: A_{2}(x ; 1)=\sigma y\right\}=\left\{y \in X: A_{2}(x ; \sigma)=y\right\}$ respectively.

It is obvious that $R(A)=\sigma y=Z\left(A_{1,2}\right)$ but $Z\left(A_{1,2}\right) \subset Z\left(A_{\sigma, 2}\right)$. Thus $X$ has a superclass partition as

(a) $X=Z(A) \oplus R\left(A_{\sigma, 2}\right)$, i.e., $X=Z(A) \cup R\left(A_{\sigma, 2}\right)$ and $Z(A) \cap R\left(A_{\sigma, 2}\right)=\emptyset$ or

(b) $X=Z(A) \oplus Z\left(\mathrm{~A}_{\sigma, 2}\right)$, i.e., $X=Z(A) \cup Z\left(\mathrm{~A}_{\sigma, 2}\right)$ and $Z(A) \cap Z\left(\mathrm{~A}_{\sigma, 2}\right)=\emptyset$.

Let $\mathbf{M}_{m n}$ be the set of all rectangular matrices of order $m \times n, \mathbf{M}_{n}$ be the set of all square matrices of order $n, \mathrm{~N}_{n}$ be the set of all non-singular matrices of order $n, \operatorname{Inv}\left(\mathrm{N}_{n}\right)$ be the set of all involutory matrices of order $n, \mathrm{~S}_{n}$ be the set of all singular matrices of order $n$ and $\mathrm{I} n$ be the set of all identity matrices of order $n$. Let $\sigma, \rho>0$. Consider the class of sets

$\operatorname{Inv}\left(\mathrm{N}_{n}\right)=\left\{A \in \mathrm{N}_{n}: A^{2}=I\right\}$ and $\operatorname{Inv}\left(\mathrm{N}_{n} ; \sigma\right)=\left\{A \in \mathrm{N}_{n}: A^{2}=\sigma I\right\}$

$\operatorname{Pr}\left(\mathrm{S}_{n}\right)=\left\{B \in \mathrm{S}_{n}: B^{2}=B\right\}$ and $\operatorname{Pr}\left(\mathrm{M}_{n} ; \rho\right)=\left\{B \in \mathrm{S}_{n}: B^{2}=\rho B\right\}$.

For $\sigma<0$, we say $A$ is skew $\sigma$-involutory or idempotent and for $\rho<0$, we say $B$ is skew $\rho$-projective.

Example: Let the function $f: \mathrm{R} \rightarrow \mathrm{R}$ be any arbitrary function.

(i) The matrix $A(x, y)=\left(\begin{array}{cc}x & 0 \\ f & -x\end{array}\right) \in \operatorname{Inv}\left(\mathrm{N}_{2} ; x^{2}\right)$ and the transpose of $A(x, y)$ is also $x^{2}$-involutory for all $x \in X$.

(ii) The matrix $\left(\begin{array}{cc}x & x \\ y-x & x\end{array}\right) \in \operatorname{Inv}\left(\mathrm{N}_{2} ; x y\right)$ and the transpose of $B(x, y)$ is also $x y$-involutory for all $x \in X$.

(iii) The matrix $C(x, y)=\left(\begin{array}{ccc}x & 0 & 0 \\ x & -x & 0 \\ x & -2 x & x\end{array}\right) \in \operatorname{Inv}\left(\mathrm{N}_{3} ; x^{2}\right)$ and the transpose of $C(x, y)$ is also $x^{2}$-involutory for all $x \in X$.

(iv) In general, the matrix $D(x, y)=\left(d_{i j}\right)$ where

$d_{i j}=\left\{\begin{array}{ll}0, & \text { if } i<j ; \\ (-1)^{i+j}\left(\begin{array}{l}i \\ j\end{array}\right) x, & \text { if } i \geq j .\end{array} \in \operatorname{Inv}\left(\mathbf{N}_{3} ; x^{n}\right)\right.$

for $1 \leq i, j \leq n$ and the transpose of $D(x, y)$ is also $x^{n}$-involutory for all $x \in X$.

\section{RESULTS/FINDINGS}

\section{The Problems:}

Let $X$ be a Hilbert space and $K \subset X, P: K \rightarrow X^{*}$ be a continuous, invertible map and non-expansive map on $K$. The concepts of involutory variational inequality problem (IVIP) and Projective variational inequality problem (PVIP) are defined as follows:

1. The $\sigma$-involutory variational inequality problem (IVIP) is to find $y \in K$ such that $\left\langle\mathrm{A}_{2}(y ; \sigma), x-y\right\rangle \geq 0$ for all $x \in K$

2. The $\rho$-projective variational inequality problem (PVIP) is to find $y \in K$ such that $\langle\mathrm{B}(y ; \rho), x-y\rangle \geq 0$ for all $x \in K$ 


\section{The Results}

The existence of the solution of the $\sigma$-IVIP and $\rho$-PVIP are established in the following theorems.

\section{Theorem}

Let $\mathrm{K}$ be a compact and convex set in the Hilbert space $\mathrm{X}$ with dual space $X^{*}=\mathrm{X}$. If the map A: $\mathrm{K} \rightarrow X^{*}$ is the continuous invertible map with respect to $\sigma \in \mathrm{R}$ and $\mathrm{A}_{\sigma, 2}$ is non-expansive, then there exists a $\mathrm{y} \in \mathrm{K}$ such that $\mathrm{y}$ solves $\sigma$-IVIP.

Proof: Since $\mathrm{K}$ is a compact and convex set in the Banach space $\mathrm{X}$ with dual space $X^{*}=X$, then for every continuous invertible map $A: K \rightarrow X$, the map A is continuous and non-expansive maps so has a fixed point $y$ in $K$ implying $0=$ $\mathbb{P}(A(y))=\mathbb{P}(A(y ; \sigma))=\mathbb{P}_{K}^{A}(y ; \sigma)$, which follows that $y$ solves the $\sigma$-IVIP of finding $y \in K$ such that $\langle y, x-y\rangle \geq \rho^{-1}\left\langle A^{2}(y), x-\right.$ $y\rangle$ for all $x \in K$, i.e., $\left\langle\left(A^{-1}-\rho^{-1} A\right)(y), x-y\right\rangle \geq 0$ for all $x \in K$. which is equivalent to the fixed point problem: Finding a fixed point $y \in K$ such that $y=\mathbb{P}_{\mathrm{K}}\left(\sigma^{-1} A^{2}(y)\right)$. This completes the proof.

\section{Theorem}

Let $K$ be a compact and convex set in the Hilbert space $X$ with dual space $X^{*}=X$. If the map $A: K \rightarrow X^{*}$ is the continuous invertible map and $A^{-1}-\sigma^{-1} A$ is non-expansive, then there exists a $y \in K$ such that y solves IVIP.

Proof. Since $K$ is a compact and convex set in the Banach space $X$ with dual space $X^{*}=X$, then for every continuous invertible map $A: K \rightarrow X^{*}$ and $F=A^{-1}-\sigma^{-1} A$, the map $I-\pi F$ is continuous and non-expansive maps, so have a fixed pointy in $\mathrm{K}$, i.e, $y=\mathbb{P}_{K}(I-\pi F)(y)$ which follows that $y$ solves the VIP

$\langle y, x-y\rangle \geq\langle(I-\pi F)(y), x-y\rangle$ for all $x \in K$, i.e.,

$\left\langle A^{-1}(y), x-y\right\rangle \geq \sigma^{-1}\langle A(y), x-y\rangle$ for all $x \in K$.

This completes the proof.

\section{DISCUSSION / ANALYSIS}

\section{Projected Dynamical System and Self involutory VIP:}

The projection operator for the involutory variational inequality problem is denoted by

$\mathbb{P}_{K}(x)=\arg \min _{y \in C}\|y-x\|$, where $C$ is the feasible space in the space $X=R^{n}$. With the help of an improved projection method developed by Solodov and Svaiter (1997), the algorithm of the involutory variational inequality problem is developed. Das, P. K., \&Baliarsingh, P. (2020), Fukushima, M. (1986), Noor, M. A. \&Rassias, T.M. (1999).

If $H_{i}$ is the hyperspace which contains the solution set $S$ of the VIP and $\partial H_{i}$ is the hyperplane which strictly separates the current approximation $x^{i}$ from any solution $x^{*}$ of the problem. The interior-point $z$ of the convex set $C$, hyperspace $H$, hyperplane $\partial H$, and the residual function $r(x)$ are defined as follows:

$$
\begin{aligned}
& H_{i}=\left\{x^{i} \in \mathrm{R}^{n}:\left\langle F\left(z^{i}\right), x^{i}-z^{i}\right\rangle \geq 0, z^{i} \in C\right\} \\
& \partial H_{i}=\left\{x^{i} \in \mathrm{R}^{n}:\left\langle F\left(z^{i}\right), x^{i}-z^{i}\right\rangle=0, z^{i} \in C\right\} \\
& r\left(x^{i}\right)=x^{i}-\mathbb{P}_{C}\left(F\left(x^{i}\right)\right) .
\end{aligned}
$$

The algorithm for the improved extragradient method is

$r\left(x^{i}\right)=x^{i}-\mathbb{P}_{C}\left(x^{i}-F\left(x^{i}\right)\right)$

$z^{i}=\left(1-t_{i}\right) x^{i}+t_{i} \mathbb{P}_{C}\left(x^{i}-F\left(x^{i}\right)\right) \in C, t_{i} \in(0,1)$,

$F\left(z^{i}\right) \in \partial H_{i}$.

\section{Algorithm or IVIP}

We define the self-solvable improved extra gradient method to solve the variational inequalities.

$$
\begin{aligned}
F\left(x^{i}\right) & =\rho^{-1} P^{2}\left(x^{i}\right) \in H_{i}, \\
r\left(x^{i}\right) & =x^{i}-\mathbb{P}_{C}\left(x^{i}-F\left(x^{i}\right)\right) \\
z^{i} & =\left(1-t_{i}\right) x^{i}+t_{i} \mathbb{P}_{C}\left(x^{i}-F\left(x^{i}\right)\right) \in C, t_{i} \in(0,1), \\
F\left(z^{i}\right) & =\rho^{-1} P^{2}\left(z^{i}\right) \in \partial H_{i} .
\end{aligned}
$$

The new iterative solution of the IVIP is

$$
\begin{aligned}
x^{i+1} & =\mathbb{P}_{C \cap H_{i}}\left(\bar{x}^{i}\right), \\
\bar{x}^{i} & =\mathbb{P}_{H_{i}}\left(x^{i}\right)=x^{i}-\frac{\left\langle F\left(z^{i}\right), x^{i}-z^{i}\right\rangle}{\left\|F\left(z^{i}\right)\right\|^{2}} F\left(z^{i}\right)=x^{i}-t_{i} \frac{\left\langle F\left(z^{i}\right), r\left(x^{i}\right)\right\rangle}{\left\|F\left(z^{i}\right)\right\|^{2}} F\left(z^{i}\right) \\
& =x^{i}-t_{i} \frac{\left\langle\rho^{-1} P\left(z^{i}\right), r\left(x^{i}\right)\right\rangle}{\left\|\rho^{-1} P\left(z^{i}\right)\right\|^{2}}\left(\rho^{-1} P\right)\left(z^{i}\right)+t_{i} \frac{\left\langle P^{-1}\left(z^{i}\right), r\left(x^{i}\right)\right\rangle}{\left\|P^{-1}\left(z^{i}\right)\right\|^{2}} P^{-1}\left(z^{i}\right) \\
& =x^{i}-t_{i} \frac{\left\langle P\left(z^{i}\right), r\left(x^{i}\right)\right\rangle}{\left\|\rho^{-1} P\left(z^{i}\right)\right\|^{2}} P\left(z^{i}\right)+t_{i} \frac{\left\langle P^{-1}\left(z^{i}\right), r\left(x^{i}\right)\right\rangle}{\left\|P^{-1}\left(z^{i}\right)\right\|^{2}} P^{-1}\left(z^{i}\right)
\end{aligned}
$$




\section{RK methods for Initial PDS involving the IVIP}

Runge-Kutta type method of order 2 for the initial value problem with a given projected dynamical system

$$
\begin{aligned}
F(x) & =\rho^{-1} P(x) \\
\frac{d x}{d t} & =\Pi_{K}\left(P^{-1}(x) ;-F(x)\right), x(0)=x_{0}
\end{aligned}
$$

is

$x_{n+1}=\mathbb{P}\left(P_{n}^{-1}(x)-t_{n} \rho^{-1} P(x)\right)$

where

$$
\begin{aligned}
P_{n}(x) & =\frac{1}{2}\left(k_{n 1}(x)+k_{n 2}(x)\right) \\
k_{n 1}(x) & =P(x) \\
k_{n 2}(x) & =\mathbb{P}\left(\rho^{-1} x_{n}-t_{n} k_{n 1}(x)\right)
\end{aligned}
$$

Hence the RK-2 type method is

$$
\begin{aligned}
x_{n+1} & =\mathbb{P}\left(x_{n}-t_{n} F_{n}(x)\right) \\
& =\mathbb{P}\left[x_{n}-\frac{t_{n}}{2}\left(k_{n 1}(x)+k_{n 2}(x)\right)\right] \\
& =\mathbb{P}\left[x_{n}-\frac{t_{n}}{2}\left(F(x)+\mathbb{P}\left(x_{n}-t_{n} F(x)\right)\right)\right]
\end{aligned}
$$

\section{RK-4 Method for Initial PDS involving the IVIP:}

Runge-Kutta type method of order 4 for the initial value problem with a given projected dynamical system

$$
\begin{aligned}
F(x) & =P^{-1}(x)-\rho^{-1} P(x) \\
\frac{d x}{d t} & =\Pi_{K}(x ;-F(x)), x(0)=x_{0}
\end{aligned}
$$

is

$$
x_{n+1}=\mathrm{P}\left(x_{n}-t_{n} F_{n}(x)\right)
$$

where

$$
\begin{aligned}
F_{n}(x) & =\frac{1}{6}\left(k_{n 1}(x)+2 k_{n 2}(x)+2 k_{n 3}(x)+k_{n 4}(x)\right) \\
k_{n 1}(x) & =F(x) \\
k_{n 2}(x) & =\mathbb{P}\left(x_{n}-\frac{t_{n}}{2} k_{n 1}(x)\right) \\
k_{n 3}(x) & =\mathbb{P}\left(x_{n}-\frac{t_{n}}{2} k_{n 2}(x)\right) \\
k_{n 4}(x) & =\mathbb{P}\left(x_{n}-t_{n} k_{n 3}(x)\right) .
\end{aligned}
$$

Hence the RK-4 method is

$$
\begin{aligned}
x_{n+1}= & \mathbb{P}\left(x_{n}-t_{n} F_{n}(x)\right) \\
= & \mathbb{P}\left[x_{n}-\frac{t_{n}}{6}\left(k_{n 1}(x)+2 k_{n 2}(x)+2 k_{n 3}(x)+k_{n 4}(x)\right)\right] \\
= & \mathbb{P}\left[x_{n}-\frac{t_{n}}{6}\left(F(x)+2 \mathbb{P}\left(x_{n}-\frac{t_{n}}{2} F(x)\right)+2 \mathbb{P}\left(x_{n}-\frac{t_{n}}{2} \mathbb{P}\left(x_{n}-\frac{t_{n}}{2} F(x)\right)\right)\right.\right. \\
& \left.\left.+\mathbb{P}\left(x_{n}-t_{n} \mathbb{P}\left(x_{n}-\frac{t_{n}}{2} \mathbb{P}\left(x_{n}-\frac{t_{n}}{2} F(x)\right)\right)\right)\right)\right]
\end{aligned}
$$




\section{CONCLUSION}

1. The concept of involutory variational inequality problems or involutory variational inequality problems are defined.

2. With the help of an improved projection method developed by Solodov and Svaiter, the algorithm of involutory variational inequality problems is studied.

3. Using the RK-4 method, the algorithm of the projected dynamical system is provided whose equilibrium point solves the involutory variational inequality problems.

\section{LIMITATION AND STUDY FORWARD}

The method used by the projection operator with more variables is critical and slow. To increase the performance of the method we can use the relaxed projection method.

\section{ACKNOWLEDGEMENT}

We are grateful to the reviewers for their valuable suggestions.

\section{AUTHORS CONTRIBUTION}

Mr. S. Mitra has done this work under the guidance of Dr. P. K. Das, the supervisor who is the co-author of this paper.

\section{REFERENCES}

1. Bardaro, C., \& Ceppitelli, R. (1988). Some further generalizations of Knaster-Kuratowski Mazurkiezicz theorem and minimax inequalities, Journal of Mathematical Analysis and Applications, 132(2), $484-490$. https://doi.org/10.1016/0022-247X(88)90076-5

2. Behera, A.,\& Panda, G. K. (1997).Variational inequality in Hausdorff topological vector space, Indian J. Pure Appl. Math, 28(2), $181-187$.

3. Behera, A., Das, P. K. (2006). Variational Inequality Problems in H-spaces, International Journal of Mathematics and Mathematical Sciences, Article 78545, 1 - 18. https://doi.org/10.1155/IJMMS/2006/78545

4. Das P. K., \& Mohanta, S. K. (2009). Generalized VIP, Generalized Vector CP in Hilbert Spaces, Riemannian $\mathrm{n}$-Manifold, $\mathbf{S}^{\mathrm{n}}$ and Ordered Topological Vector Spaces: A Study Using Fixed Point Theorem and Homotopy Function, Advances in Nonlinear Variational Inequalities, 12(2), 37 - 48.

5. Das, P.K. (2011). An iterative method for (AGDDVIP) in Hilbert space and the Homology theory to study the $\left(\mathrm{GDDCP}_{\mathrm{n}}\right)$ in Riemannian n-manifolds in the presence of fixed point inclusion, European Journal of Pure and Applied Mathematics, 4(4), 340-360.

6. Das, P. K., \&Baliarsingh, P. (2020). Involutory difference operator and $\mathrm{j}^{\text {th }}$-difference operator, PanAmerican Mathematical Journal, 30(4), $53-62$.

7. Fang, C., Chen S., \& Zheng, J. (2013). A projection-type method for multivalued variational inequality, Abstract and Advanced Analysis, Article ID 836720. https://doi.org/10.1155/2013/836720

8. Fukushima, M. (1986). A relaxed projection method for variational inequalities, Mathematical Programming, 35, 58 - 70. https://doi.org/10.1007/BF01589441

9. Giannessi, F. (1980).Vector variational inequalities and vector equilibria, Wiley, New York.

10. He, B. S., \& Liao, L. Z. (2002). Improvements of some projection methods for monotone nonlinear variational inequalities. Journal of Optimization Theory and applications, 112(1), 111-128. https://doi.org/10.1023/A:1013096613105

11. Lee, G. M., Kim, D. S., \& Lee, B. S. (1996). Generalized vector variational inequality. Applied Mathematics Letters, 9(1), 39-42. https://doi.org/10.1016/0893-9659(95)00099-2

12. Limaye, B. V. (1997). Functional analysis, New Age International (P) Limited, New Delhi.

13. Malitsky, Y. (2015). Projected reflected gradient methods for monotone variational inequalities. SIAM Journal on Optimization, 25(1), 502-520. https://doi.org/10.1137/14097238X

14. Mosco, U. (1972). Dual variational inequalities. Journal of Mathematical Analysis and Applications, 40(1), 202-206. https://doi.org/10.1016/0022-247X(72)90043-1

15. Nagurney, A., \&Zhang, D. (1996). Projected dynamical systems and variational inequalities with applications, Springer Science. https://doi.org/10.1007/978-1-4615-2301-7

16. Noor, M. A. \&Rassias, T.M. (1999). Projection Methods for monotone variational inequalities, Journal of Mathematical Analysis and Applications, 237(2), 405- 412. https://doi.org/10.1006/jmaa.1999.6422

17. Siddiqi, A. H., Ansari, Q. H., \& Khaliq, A. (1995). On vector variational inequalities. Journal of Optimization Theory and Applications, 84(1), 171-180. https://doi.org/10.1007/BF02191741

18. Solodov, M. V., \&Svaiter, B. F. (1997). A new projection method for variational inequality problems, SIAM J. Cont. Optim. 37(3), 765 - 776. https://doi.org/10.1137/S0363012997317475

19. Stampacchia, G. (1964). Formesbilineairescoercitives sur les ensembles convexes, Comptesrendushebdomadaires des sances de l'Acadmie des sciences, 258, 4413-4416. 
International Journal of Students' Research in Technology \& Management elSSN: 2321-2543, Vol 9, No 2, 2021, pp 18-24

https://doi.org/10.18510/ijsrtm.2021.924

20. Thong, D. V., \& Cho, Y. J. (2020). New strong convergence theorem of the inertial projection and contraction method for variational inequality problems. Numerical Algorithms, 84(1), 285-305. https://doi.org/10.1007/s11075-019-00755-1 